\title{
Design of a Multipurpose Radio Controlled Surveillance Vehicle to Monitor Risky Areas
}

\author{
https://doi.org/10.3991/ijes.v7i2.10380 \\ Mobasshir Mahbub \\ East West University, Dhaka, Bangladesh \\ mbsrmhb@gmail.com
}

\begin{abstract}
Wireless technology is one of the most common technologies used in our day to day life. As wireless technology is very much easier to implement rather than wired, day by day its application is increasing. Through the continuous development of wireless communication technology it is now highly used in wireless equipment controlling. That is we can control our electronics and electrical equipments from a certain distant part. In this paper a multipurpose radio controlled car is designed with nRF24L01 wireless transceiver module, Arduino Uno R3 and DC motors to monitor risky areas. The RC vehicle is controlled by a distant controller or transmitter unit to move the vehicle to the risky location for monitoring.
\end{abstract}

Keywords-RC vehicle; Wireless communication; Wireless controller; Microcontroller; Arduino; nRF24L01.

\section{Introduction}

The nRF24L01 wireless transceiver module controlled with Arduino MCU can create a more flexible, low cost and user friendly system [1]. Programming of this system is also easier comparative to other MCU's [2]. Using this system a radio controlled car can be built to monitor risky areas and situation where human presence is not possible or highly risky. The vehicle is named as multipurpose vehicle because we can use it in multiple ways such as we can use it for video transmission by simply adding a video transmitter in it. For example, an area like a small tunnel where we are unable to go but it is possible to send a small radio controlled vehicle equipped with a video transmitter to see the scenario of the tunnel. Another example can be determination of the condition of a toxic area where hazardous gases are present and human presence can be harmful but we can send the vehicle equipped with gas sensors to find out and measure the amount of the gases in the area. This is only possible through the radio controlled vehicle. As all these are risky conditions and a small radio control vehicle can easily move there and grab the data. 


\section{System Architecture and Design}

\subsection{Working principle}

At the transmitter unit, the controller modules attached with the controller MCU that is Arduino will receive control signals. Then it will process each corresponding signal to determine the particular signals for the receiving ends equipments. After that the corresponding signal will be passed to the nRF24L01 wireless transceiver module to transmit [6].

At the receiver unit, the receiver's nRF24L01 will receive each signal and will pass to the MCU. Then the MCU that is

Arduino will process and analyze the signals to determine the corresponding signal for particular equipment and will execute the instruction that is sent from the controller [3].

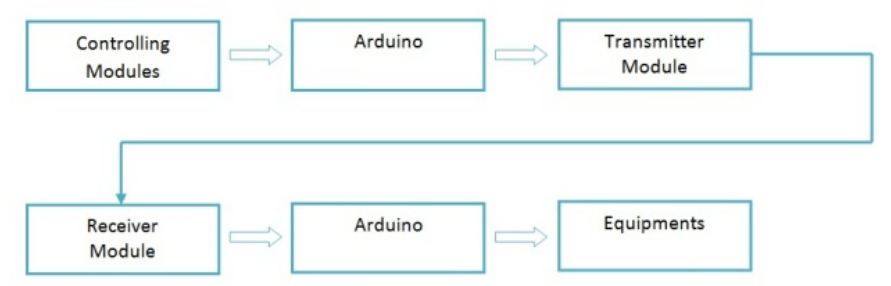

Fig. 1. Overview of the System

\subsection{Required components}

\section{Components in transmitter unit}

- Arduino UNO R3 (MCU)

- nRF24L01 Wireless Transceiver Module

- 2x Thumb Joystick Module

- 2x Push Button

- Breadboard

- Wires (Male to Male, Male to Female)

Components required at receiver to control DC motor of a multipurpose $\mathrm{RC}$ vehicle

- Arduino UNO R3 or Mega 2560

- nRF24L01 Wireless Transceiver Module

- 4x DC motor

- Wires (Male to Male, Male to Female) 


\subsection{Description of major components}

This section will provide an overview of required components. Short description of their working procedures, schematic of components and graphic image of those components will be in this section.

Arduino UNO R3: Arduino Uno is a microcontroller unit based on the ATmega328P chip. It has 14 digital input/output pins (among 6 can be used as PWM outputs), 6 analog inputs, a $16 \mathrm{MHz}$ quartz crystal, a USB connection, a power jack, an ICSP header and a reset button. This contains everything onboard needed to support the microcontroller unit; only needed to connect it to a computer with a USB cable or power it with an AC-to-DC adapter or battery to power up [1].

The "Uno" means one in Italian language and was designated to indicate the release of Arduino IDE 1.0 [2]. The newly developed Arduino Uno board and the first version of IDE, version 1.0 were the reference versions. The Uno board is the first in a series of USB Arduino boards, and the reference model for the Arduino platform [1].

Table 1. Technical specification

\begin{tabular}{|c|l|}
\hline Microcontroller & ATmega328P \\
\hline Operating Voltage & $5 \mathrm{~V}$ \\
\hline Input Voltage (recommended) & $7-12 \mathrm{~V}$ \\
\hline Input Voltage (limit) & $6-20 \mathrm{~V}$ \\
\hline Digital I/O Pins & 14 (of which 6 provide PWM output) \\
\hline PWM Digital I/O Pins & 6 \\
\hline Analog Input Pins & 6 \\
\hline DC Current per I/O Pin & $20 \mathrm{~mA}$ \\
\hline DC Current for 3.3V Pin & $50 \mathrm{~mA}$ \\
\hline Flash Memory & $32 \mathrm{~KB}(\mathrm{ATmega328P})$ of which $0.5 \mathrm{~KB}$ used by bootloader \\
\hline SRAM & $2 \mathrm{~KB}(\mathrm{ATmega328 \textrm {P }})$ \\
\hline EEPROM & $1 \mathrm{~KB}(\mathrm{ATmega328 \textrm {P }})$ \\
\hline Clock Speed & $16 \mathrm{MHz}$ \\
\hline LED_BUILTIN & 13 \\
\hline Length & $68.6 \mathrm{~mm}$ \\
\hline Width & $53.4 \mathrm{~mm}$ \\
\hline Weight & $25 \mathrm{~g}$ \\
\hline
\end{tabular}




\section{Schematic}

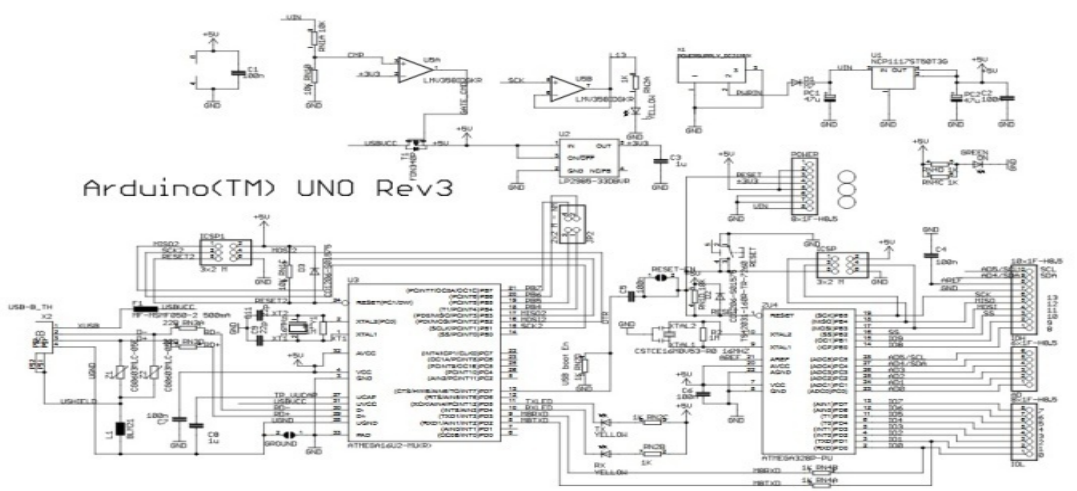

Fig. 2. The schematic diagram of Arduino Uno board

\section{Pinout}

\section{ATMEGA328 PINOUT}

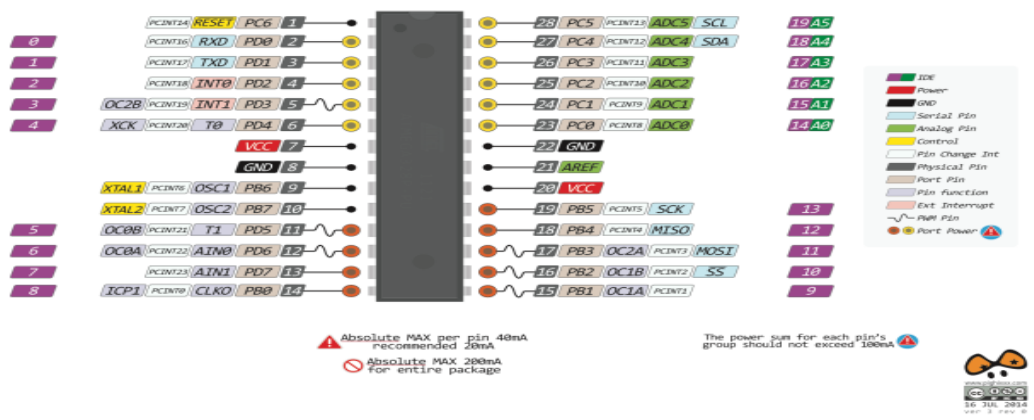

Fig. 3. Pinout of ATMEGA328P

\section{UNOPINOUT}

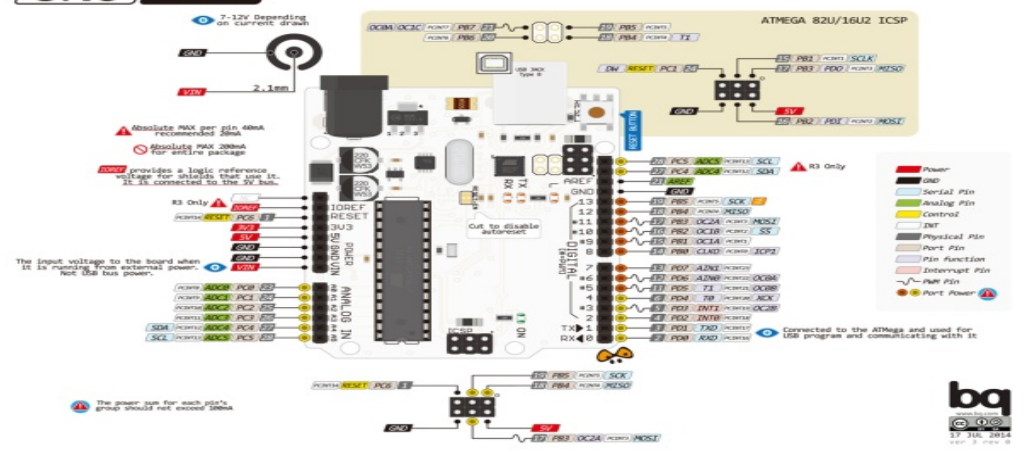

Fig. 4. Pinout of Arduino Uno 
nRF24L01 Wireless transceiver module: The nRF24L01 module is a single chip 2.4GHz transceiver having embedded baseband protocol engine (Enhanced ShockBurst $^{\mathrm{TM}}$ ), specially designed to provide support to ultra low power wireless applications. The nRF24L01 is designed for operation ISM frequency band at 2.400 $2.4835 \mathrm{GHz}$. An MCU and few external passive components are required to design a radio communication system with the $\mathrm{nRF} 24 \mathrm{~L} 01$.

The nRF24L01 module is designed to be configured and operated through a Serial Peripheral Interface. This interface has the availability of register map. Register map contains all configuration registers and is accessible in all operation modes of nRF24L01 module.

The embedded baseband protocol engine is based on packet communication. It supports various modes such as manual operation, advanced autonomous protocol operation etc. Internal FIFOs provide a smooth data stream between the radio front end and the MCU. The Enhanced Shock-

Burst $^{\mathrm{TM}}$ engine reduces system cost by controlling all the high-speed link layer operations.

The radio front end of nRF24L01 module uses GFSK modulation technique. The module supports user configurable parameters such as air data rate and frequency channel output power.

The air data rate supported by the module is configurable up to $2 \mathrm{Mbps}$. The high data rates and two power saving modes combined makes the nRF24L01 module very compatible for ultra low power system designs.

High Power Supply Rejection Ratio (PSRR) and a wide power supply range are ensured by internal voltage regulators [4].

Features of nRF $24 L 01$

- $2.4 \mathrm{GHz} \mathrm{RF}$ transceiver Module

- Operating Voltage: $3.3 \mathrm{~V}$

- Nominal current: $50 \mathrm{~mA}$

- Range: $50-200$ feet

- Operating current: 250mA (maximum)

- Communication Protocol: SPI

- Baud Rate: 250 kbps - 2 Mbps.

- Channel Range: 125

- Maximum Pipelines/node: 6

- Low cost wireless solution [5]

\section{Schematic}




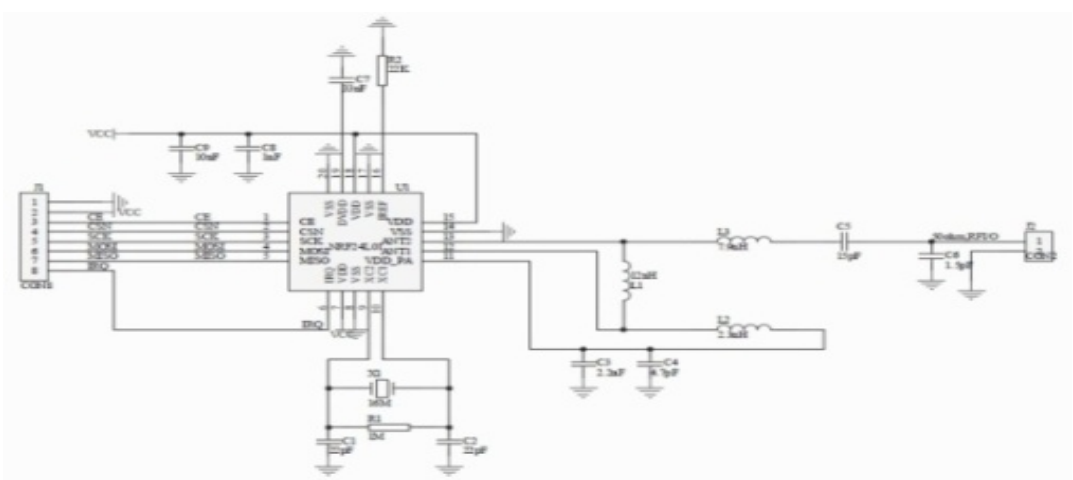

Fig. 5. Schematic diagram of nRF24L01

\section{Pinout}

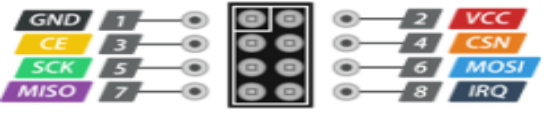

nRF24LO1+ Pinout

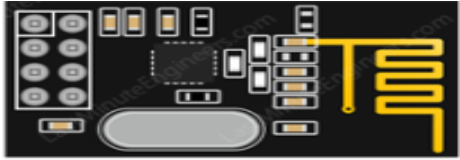

Last Minute

Fig. 6. Pinout of nRF24L01

Joystick module: In electronics there are many applications of Joystick. This module mostly used in Arduino based DIY projects and Robotic Controlling. This module provides analog output that is why it can be applied for feeding the analog input depending on direction or movement.

Joystick Module can be used with Arduino, Raspberry Pi and any other MCUs. Simply we have to connect the $\mathrm{X}$ and $\mathrm{Y}$ axis corresponding axis Pins VRx and VRy to the ADC Pins of the MCU. If it needed to be uses as a switch then have to connect it to the digital Pin of the MCU [6].

\section{Technical specification}

- Two independent potentiometer for each of $\mathrm{X}$ and $\mathrm{Y}$ axis.

- Auto return to center position

- Operating Voltage: 5V

- Internal Potentiometer value: $10 \mathrm{k}$

- $2.54 \mathrm{~mm}$ pin interface leads

- Dimensions: 1.57 in x 1.02 in x 1.26 in $(4.0 \mathrm{~cm}$ x $2.6 \mathrm{~cm}$ x $3.2 \mathrm{~cm})$

- Operating temperature: 0 to $70{ }^{\circ} \mathrm{C}$ 


\section{Schematic}

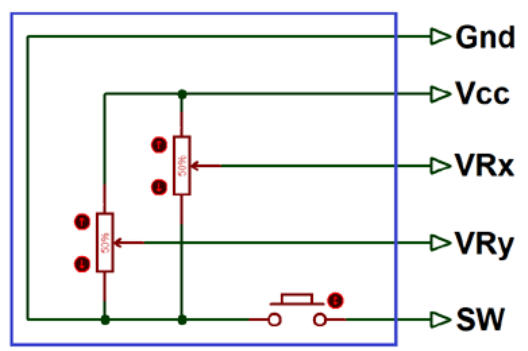

Fig. 7. Schematic diagram of joystick module

\section{Pinout}
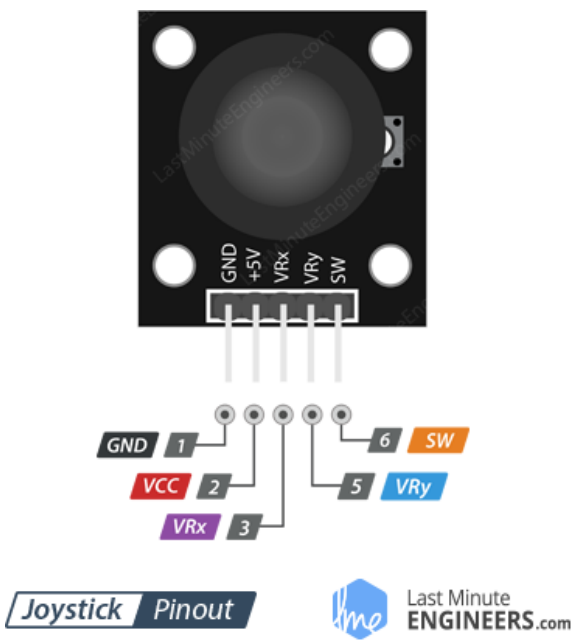

Fig. 8. Pinout of joystick module

Push button - Tactile switch: Push-Button is a normally-open tactile switch. It allows us to power the circuit or make any particular connection only when the button is pressed. Simply to be said, it leads the circuit connected when it is pressed and breaks when it gets released. A push button can also be used to trigger the SCR by gate terminal. It can also be used to feed the analog or digital input system of MCU to direct the MCU for a desired decision making. It is one of the most common buttons which is used in our daily life electronic components. Its application includes use in calculators, push-button telephones, kitchen appliances, magnetic locks and various mechanical and electronic appliances in both home and commercials. 


\section{Technical specification}

- Mode of Operation: Tactile feedback

- Power Rating: MAX 50mA 24V DC

- Insulation Resistance: $100 \mathrm{Mohm}$ at $100 \mathrm{v}$

- Operating Force: $2.55 \pm 0.69 \mathrm{~N}$

- Contact Resistance: MAX 100mOhm

- Operating Temperature: -20 to $+70{ }^{\circ} \mathrm{C}$

- Storage Temperature: -20 to $+70^{\circ} \mathrm{C}[9]$

\section{Schematic}

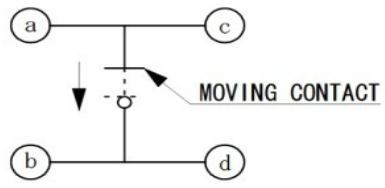

Fig. 9. Schematic diagram of push button

\section{Pinout}

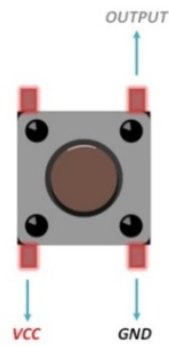

Fig. 10.Pinout of push button

DC motor: A DC motor is a kind of rotary electrical machine that converts the direct current electrical energy into mechanical energy. The most common type is based on the forces generated by magnetic fields. DC motors have some internal mechanism, either electromechanical or electronic. This periodically changes the rotating direction of current flow in part of the DC motor.

DC motors have different voltage and current ratings. But in case of $\mathrm{MCU}$, the motors ranging from $4.5 \mathrm{v}$ to $12 \mathrm{v}$ is more suitable to the MCU. Up to $24 \mathrm{v}$ can also be used with MCU.

To start the DC motor's rotation just connect the positive $(+)$ side of battery to one terminal and the negative (-) to the other terminal and the motor should be rotating. To reverse the rotations of the motor simply interchange the terminals and the rotating will be in reversed direction.

The application includes windmill projects, basic Electronics projects and as Robot wheels etc [7]. 


\section{Schematic and pinout}

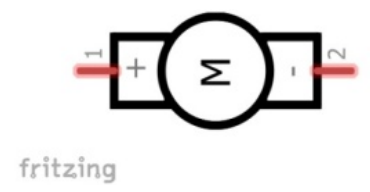

Fig. 11.Schematic diagram and pinout of a DC motor

\section{Design and implementation}

- Transmitter or controller unit

It is consisted of an Arduino Uno as MCU, nRF24L01 as control signal transmitter, 2 joystick modules and 2 push buttons to take control directions. Joysticks are used to move the vehicle and push buttons are used to trigger equipments like light, horn etc. if used. The unit can be powered up using pc USB cable by connecting with MCU's USB port and batteries ranging from $5 \mathrm{v}-20 \mathrm{v}$. The recommended supply input is $5 \mathrm{v}-$ $12 \mathrm{v}[1]$.

\section{Breadboard implementation}

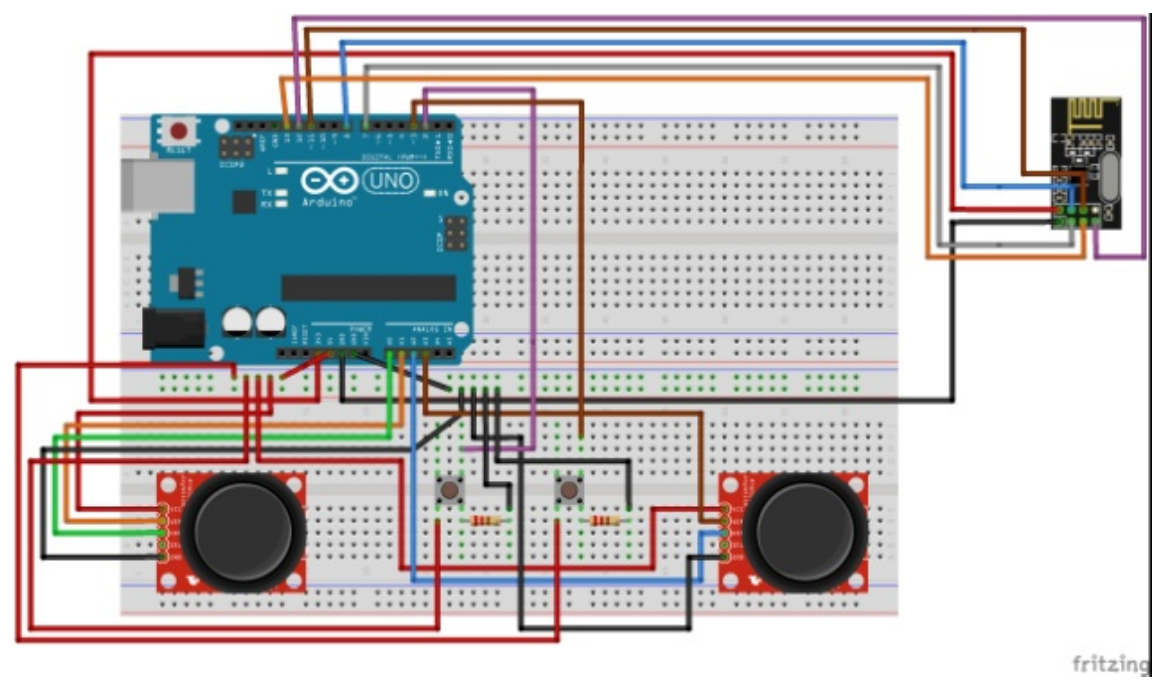

Fig. 12.Breadboard implementation of transmitter unit 


\section{Schematic}

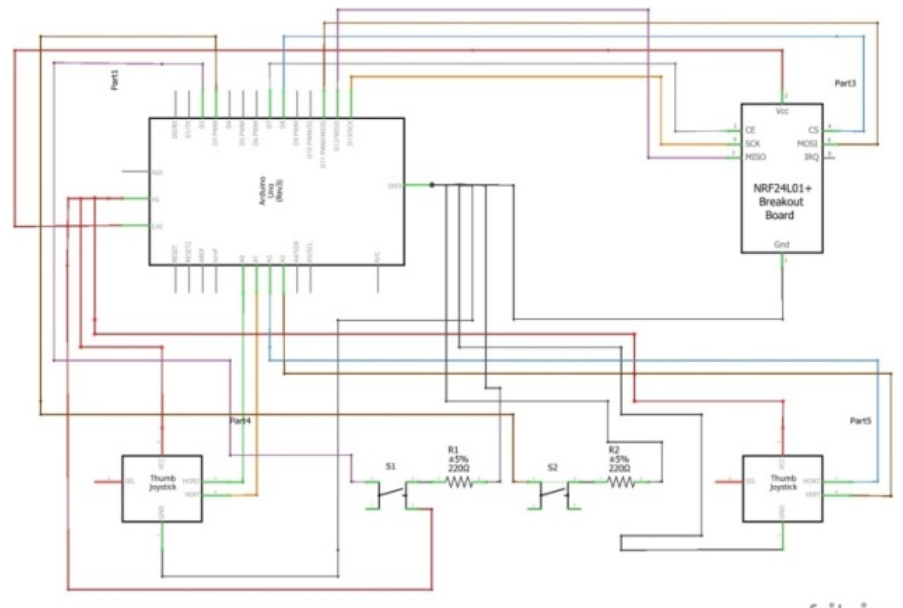

Fig. 13.Schematic diagram of transmitter unit

ii) Design of multipurpose $R C$ vehicle: Connecting four DC motors with the receiver unit according to the proper connection scheme we can make a multipurpose radio controlled vehicle [8]. I have used 4WD car chassis to implement the circuit of the vehicle below. The vehicle is to be equipped with the monitoring equipments to make it as a surveillance vehicle to monitor the risky areas. It can be equipped with a video transmitter to visualize a risky area or monitor that area where human presence is not possible. Also it can be equipped with multiple sensors like ultrasonic sensor to avoid obstacle or it can be equipped with gas sensors to monitor a high risk area where hazardous gases are present. But in this design I have designed the car only and all those equipments can be implemented on this car easily.

\section{Breadboard implementation}

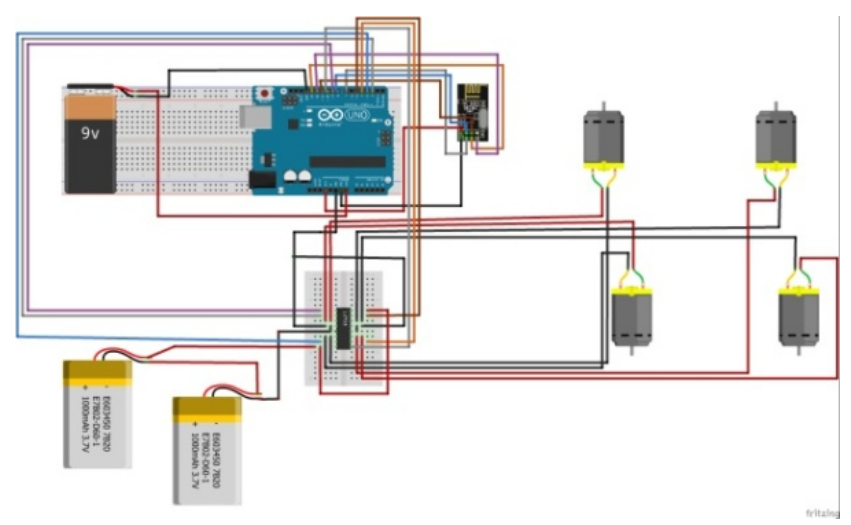

Fig. 14.Breadboard implementation of a multipurpose RC vehicle with DC motor 


\section{Schematic}

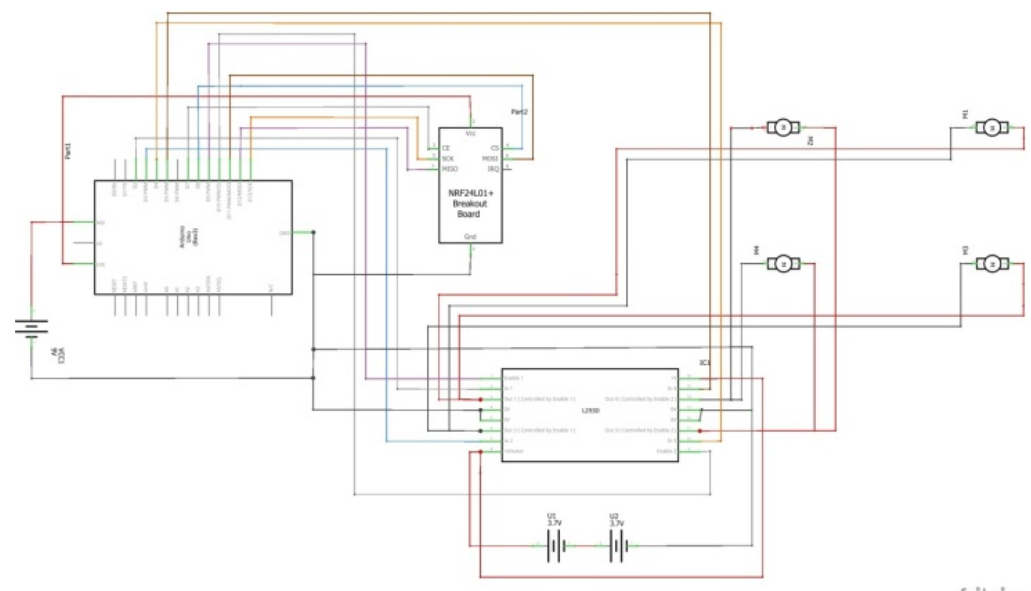

Fig. 15.Schematic diagram of a multipurpose RC vehicle with DC motor

E. MCU programming: Sketch compiler is the trademark compiler of Arduino to write the necessary codes and inject in Arduino boards or MCUs. The following programming flow charts are made based on this Sketch compiler [2].

Programming flow chart for transmitter

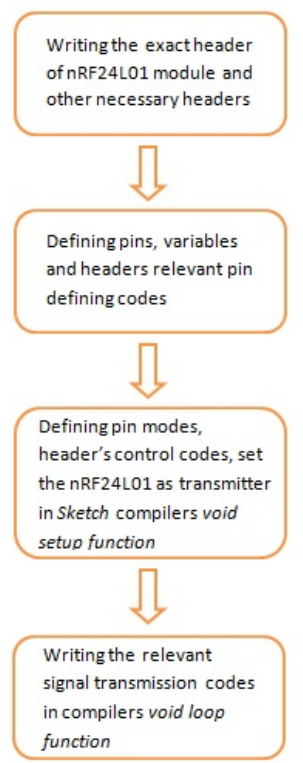

Fig. 16.Programming flow chart of transmitter 


\section{Programming flow chart for receiver}

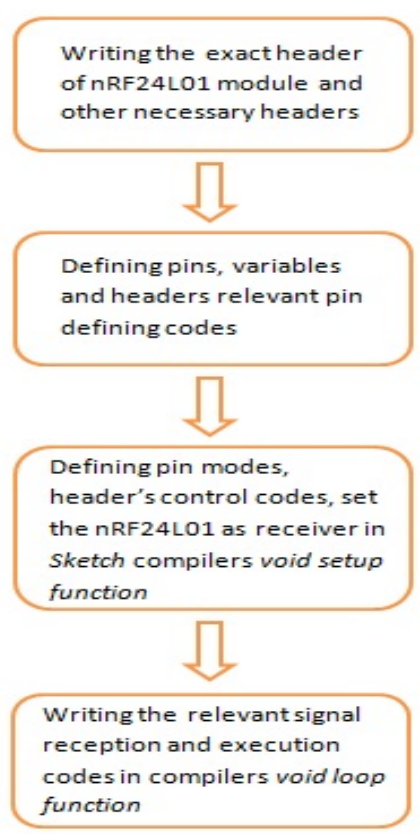

Fig. 17.Programming flow chart of receiver

\section{Discussions}

A wireless video transmitter is needed to control the movement of the vehicle towards the risky place. Through a display by observing video transmitted by the transmitter equipped in the vehicle user can operate this vehicle to move it in the desired place and can visualize and monitor the conditions of the place. If sensors are attached with the vehicle than the data collected by the components and sensors can be monitored and visualized using the serial monitor of Sketch.

\section{Conclusion}

With this vehicle a dangerous or risky area can be observed from a distant safe region within the range of the nRF24L01 transceiver module. The developed RC vehicle can be equipped with necessary components to monitor the risky environment with a low cost. This is the advantage of using this car. More research and tests can be performed to develop the vehicle to find out more and more applications of it. The building materials of this vehicle are very much cheap. An individual can easily build it and can perform research to make it more effective and to make it work according to his/her desire if he/she has the prior relevant technical knowledge. 


\section{$5 \quad$ References}

[1] Steven Barret, "Getting Started" in Arduino Microcontroller: Processing for Everyone!, 2nd ed., San Rafael, California, USA: Morgan \& Claypool, 2012, ch. 1, pp. 1-22.

[2] Steven Barret, "Programming" in Arduino Microcontroller: Processing for Everyone!, 2nd ed., San Rafael, California, USA: Morgan \& Claypool, 2012, ch. 2, pp. 23-52.

[3] S. A. Ram, N. Siddarth, N. Manjula, K. Rogan, K. Srinivasan, "Real-time Automation System Using Arduino", 2017 International Conference on Innovations in information Embedded and Communication Systems (ICIIECS), Coimbatore, India, March, 2017. https://doi.org/10.1109/iciiecs.2017.8275845

[4] Y. Wang1, C. Hu, Z. Feng1, Y. Ren1, "Wireless Transmission Module Comparison", 2014 IEEE International Conference on Information and Automation (ICIA), Hailar, China, July, 2014. https://doi.org/10.1109/icinfa.2014.6932780

[5] D. Hu1, H. Ke, W. Fu, "Research and design of control system based on NRF24101 for intellectualized vehicle", 2017 6th Data Driven Control and Learning Systems (DDCLS), Chongqing, China, May, 2017. https://doi.org/10.1109/ddcls.2017.8068155

[6] D. Ding, R. A. Cooper, D. Spaeth, "Optimized Joystick Controller", Proceedings of the 26th Annual International Conference of the IEEE EMBS, San Francisco, CA, USA, September, 2004. https://doi.org/10.1109/iembs.2004.1404350

[7] Adafruit, Toy DC Motor Datasheet. [Online]. Available: https://components101.com/sites/default/files/compocomp_datasheet/Toy\%20DC\%20mot or\%20Datasheet.pdf. [Accessed: February 7, 2019]

[8] M. A. Ahmad, K. Kishor, P. Rai, "Speed Control of a DC Motor Using Controllers", Automation, Control and Intelligent Systems, Special Issue: Impact of Gesture Recognition in the Technological Era, Vol. 2, No. 6-1, 2014, pp. 1-9. https://doi.org/10.11648/j.acis.s.2014020601.11

[9] HDK, Tactile Switches Datasheet. [Online]. Available: https://components101.com/sites/default/files/component_datasheet/Push-Button.pdf. [Accessed: February 7, 2019]

\section{Author}

Mobasshir Mahbub works for Department of Electronics and Communication Engineering, in East West University, Dhaka, Bangladesh. He is accessible over mail in mbsrmhb@gmail.com

Article submitted 2019-03-01. Resubmitted 2019-05-20. Final acceptance 2019-05-21. Final version published as submitted by the authors. 